\title{
Chitosan-alginate microparticulate delivery system for an alternative route of administration of $B C G$ vaccine
}

\author{
Liliana Aranha Caetano ${ }^{1,2}$, Rita Amaral ${ }^{2}$, Lara Figueiredo ${ }^{2}$, António J. Almeida ${ }^{2}$, Lídia M. D. Gonçalves ${ }^{2}$ \\ ${ }^{1}$ Escola Superior de Tecnologia da Saúde de Lisboa, Instituto Politécnico de Lisboa. Lisboa, Portugal \\ ${ }^{2}$ Faculdade de Farmácia, Universidade de Lisboa iMed.UL, Lisboa, Portugal; (lacaetano@ff.ul.pt)
}

\begin{abstract}
Immunisation against $M$. tuberculosis with current available BCG vaccine lacks efficacy in preventing adult pulmonary tuberculosis. Targeting nasal mucosa is an attractive option for a more effective immunization. The delivery of BCG via the intranasal route involves overcoming barriers such as crossing the physical barrier imposed by the mucus layer and ciliar remotion, cellular uptake and intracellular trafficking by antigen presenting cells. Due to its biodegradable, immunogenic and mucoadhesive properties, chitosan particulate delivery systems can act both as vaccine carrier and adjuvant, improving the elicited immune response. In this study, different combinations of Chitosan/Alginate/TPP microparticles with BCG were produced as vaccine systems. The developed microparticle system successfully modulates BCG surface physicochemical properties and promotes effective intracellular uptake by human macrophage cell lines Preliminary immune responses were evaluated after s.c. and intranasal immunisation of BALB/c mice. BCG vaccination successfully stimulated the segregation of IgG2a and IgG1, where intranasal immunisation with chitosan/alginate particulate system efficiently elicited a more equilibrated cellular/humoral immune response.
\end{abstract}

Keywords- BCG, microencapsulation, chitosan, mucosal immunization

\section{INTRODUCTION}

Tuberculosis remains an important cause of morbidity and mortality worldwide. [1] The current vaccine, Mycobacterium bovis bacillus Calmette-Guérin (BCG), has variable protective efficacy ranging from 0 to $85 \%$ in different studies [2], hence, efforts are being made in order to develop an improved vaccine. As for many pathogens, M. tuberculosis infection occurs through mucosa. Therefore, the development of an efficient vaccine will require an appropriate antigen presentation and adjuvant effect that can induce both mucosal and systemic immune response, preventing not only infectious disease but also colonization of mucosal surfaces [3].

Biodegradable particulate delivery systems have been successfully used in recent vaccine development, and mucosal delivery systems designed to enhance the immune response following mucosal immunization have been evaluated for efficacy in tuberculosis vaccines [4-5]. However, to our knowledge, none using live viable vaccines.

Live vaccines, such as BCG, provide several important advantages over other strategies, as they act like the natural infection, being readily uptaken by specialized antigen presenting cells, thus, inducing strong humoral and cellular immune responses.

As a nasal carrier system, chitosan microparticles are ideal, due to their mucoadhesive and adjuvant properties, and ability to act as antigen depot, enabling a better interaction between the carried vaccine and the membrane epithelium, thus, allowing a more efficient uptake by alveolar macrophages [6]. Through modification of particle features, such as size, zeta potential and composition, a different pattern of MHC presentation and acquired immunity can be obtained, thus, tuning and improving the immune response [7-9].

\section{GOALS}

A particle-mediated delivery system of chitosan and alginate was designed via a mild ionotropic gelation procedure with sodium tripolyphosphate (TPP), as previously described [10], for efficient encapsulation of live attenuated BCG. Physico-chemical characterization of particulate delivery system and assessment of intracellular uptake by macrophages were performed.

The titres of IgG2a and IgG1 were investigated using a purified protein derivative (PPD) bovine tuberculin, probed with serum samples collected at biweekly intervals from $\mathrm{BALB} / \mathrm{c}$ mice, following vaccination with different BCG microparticle formulations, both intranasal (i.n.) and subcutaneously (s.c.) administrated.

\section{MATERIALS AND METHODS}

\section{A. Materials}

Chitosan of different molecular weight (low molecular weight (CH-LMW, $150 \mathrm{kDa})$, medium molecular weight $(\mathrm{CH}-$ MMW, $450 \mathrm{kDa}$ ), and high molecular weight (CH-HMW, 600 $\mathrm{kDa})$ ), sodium alginate with different viscosities, and TPP were obtained from Sigma Aldrich, UK.

Both BCG strains used in this study - M. bovis BCG Pasteur (ATCC35734) and a recombinant M. bovis BCG harbouring a pMN437 plasmid [11], were kindly supplied by Prof Elsa Anes (FFUL). All cell culture reagents were from Invitrogen, UK.

\footnotetext{
$3^{\text {rd }}$ Portuguese Meeting in Bioengineering, February 2013
}

Portuguese chapter of IEEE EMBS 